\title{
Trafo Merkezleri İçin Nesnelerin İnterneti Tabanlı Kontrol Sistemi Tasarımı ve Uygulaması
}

\author{
Sercan Aygün ${ }^{1}$ Abdullah Sevin ${ }^{2}$ \\ ${ }^{1}$ Sakarya Üniversitesi, Bilgisayar ve Bilişim Mühendisliği Fakültesi, Bilgisayar ve Bilişim Mühendisliği Bölümü, Sakarya, Türkiye (ORCID: 0000-0001-6242-0391), \\ sercan.aygun@ogr.sakarya.edu.tr \\ ${ }^{2}$ Sakarya Üniversitesi, Bilgisayar ve Bilişim Mühendisliği Fakültesi, Bilgisayar ve Bilişim Mühendisliği Bölümü, Sakarya, Türkiye (ORCID: 0000-0001-9694-0870), \\ asevin@sakarya.edu.tr
}

(International Symposium on Multidisciplinary Studies and Innovative Technologies (ISMSIT) 2021 - 21-23 October 2021)

(DOI: $10.31590 /$ ejosat.1010748)

ATIF/REFERENCE: Aygün, S., Sevin, A. (2021). Trafo Merkezleri İçin Nesnelerin İnterneti Tabanlı Kontrol Sistemi Tasarımı ve Uygulaması. Avrupa Bilim ve Teknoloji Dergisi, (29), 30-35.

Öz

Elektrik enerjisinin ev ve işyerleri gibi alanlar için uygun gerilim seviyesine dönüştürülmesi trafo merkezleri tarafindan sağlanmaktadır. Trafo merkezleri ile önemli bir miktarda enerji iletimi yapılmaktadır. Günümüzde neredeyse tüm kamu ve özel kurum ve kuruluşlara ait kritik altyapılar bu elektrik iletimine bağlı olarak çalışmaktadır. Hastaneler, sanayi kuruluşları, nükleer ve diğer santraller, bankalar, askeri birimler vs. gibi birçok alandaki bu kritik altyapıda oluşabilecek sıkıntılar hayati veya maddi problemlere yol açacaktır. $\mathrm{Bu}$ nedenle bu merkezlerde meydana gelen bir arızanın hızlı bir şekilde tespit edilmesi, ilgili kişilere bildirilmesi ve arıza türüne göre doğru müdahalenin yapılması hayati ve maddi kayıpların önüne geçecektir. Trafo merkezi kontrolünde uzun süre kullanılan SCADA sistemleri güvenli bir seçenek olmasının yanı sıra pahalı bir çözüm sunmaktadır. Nesnelerin interneti teknolojisinin gelişmesi ile birlikte bu soruna etkin ve daha az maliyetli çözümler üretilmeye başlanmıştır. Bu makalede trafo merkezlerinin uzaktan izlenmesini ve kontrol edilmesini sağlamak amacıyla nesnelerin interneti ve gerçek zamanlı işletim sistemi teknolojilerini kullanan alternatif bir çözüm önerisi sunulmaktadır. Önerilen sistem, ESP32 geliştirme kiti ile Blynk uygulaması üzerinden izleme ve kontrol yapılmasını sağlamaktadır. Tasarlanan sistem sayesinde yüksek gerilim durumlarına, yă̆ seviyesi arızası, yüksek sıcaklık ve aşırı yük veya akım gibi problemlere gerçek zamanlı müdahale edilebilmektedir. Daha önce kullanılan sistemlerde bulunan özelliklerin yanı sıra gerçek zamanlı işletim sisteminin kullanılması, öncelik mekanizması, redresör grubu takibi, Bluetooth gibi farklı haberleşme teknolojilerin eklenmesi ve özellikle maliyet etkin bir çözüm önerisi olması sistemin öne çıkan özellikleri olarak sunulmaktadır. Bunun yanı sıra önerilen sistemin, nesnelerin interneti teknolojisinin uygulama alanlarından biri olan akıllı şehirlerde kullanılan akıllı lambalar ile entegre bir senaryo ile de modellemesi yapılmıştır.

Anahtar Kelimeler: Nesnelerin İnterneti, Gerçek Zamanlı İşletim Sistemi, Trafo, İzleme

\section{Design and Implementation of the Internet of Things Based Control System for Transformer Centers}

\begin{abstract}
The substations provide conversion of electrical energy to the appropriate voltage level for homes and workplaces. A significant amount of energy is transmitted through these substations. Today, critical infrastructures of almost all public and private institutions and organizations operate depending on this electricity transmission. Problems in this critical infrastructure in many areas such as hospitals, industrial establishments, nuclear and other power plants, banks, military, etc., will lead to vital or financial problems. For this reason, rapid detection of a malfunction in these centers, notifying the relevant persons, and making the proper intervention according to the type of malfunction will prevent vital or financial losses. SCADA systems have been used for a long time in substation control, offer an expensive solution, and be a safe option. Effective and less costly solutions have begun to be produced with the development of the internet of things. This article presents a solution proposal that uses the internet of things and real-time operating system technologies to enable remote monitoring and control substations. The proposed system provides monitoring and control over the Blynk application with the ESP32 development kit. The designed system can be intervened in real-time problems such as high voltage situations, oil level failure, high temperature, and overload or current. In addition to the features found in previously used systems, the addition of different communication technologies such as Bluetooth, using of real-time operating system,

priority mechanism, battery monitoring, and especially a cost-effective solution offer is presented as the outstanding features of the system. In addition, the proposed system has been modeled with an integrated scenario with smart lamps used in smart cities, which is one of the application areas of the internet of things technology.
\end{abstract}

Keywords: Internet of Things, Real-Time Operating System, Substation, Monitoring

${ }^{1}$ Sorumlu Yazar: sercan.aygun@ogr.sakarya.edu.tr

http://dergipark.gov.tr/ejosat 


\section{Giriş}

Elektrik enerjisi, çeşitli enerji kaynaklarından dönüştürülerek uzun mesafeler katettikten sonra ev ve iş yerlerine gelmektedir. Üretilen enerji yüksek gerilim seviyelerindedir ve bu enerji çeşitli aşamalardan geçerek evlerde kullandığımız gerilim seviyesine dönüştürülmektedir. Trafo merkezleri, bu gerilim dönüştürme işleminin yanı sıra günlük yaşantımızda önemli bir yer tutan elektrik enerjisinin sağlıklı ve verimli dağıtımından ve iletiminden sorumludur. Trafo merkezlerinin sağlıklı işlemesi hayati ve maddi kayıpların önlenebilmesi için büyük bir öneme sahiptir.

Trafoların uzun süre kullanılabilmesi için çeşitli etkenlere karşı takibinin yapılması amacıyla SCADA (Supervisory Control and Data Acquisition) sistemleri kullanılmaktadır (Peharda vd., 2017; Tom \& Sankaranarayanan, 2017; Wester vd., 2015). Bu sistemle veriler internet üzerinden merkezi bir yerde toplanmaktadır. Ancak; bu sistem pahalı olması sebebiyle her trafo merkezinde kullanılamamaktadır (SCADA). Herhangi bir uzaktan izleme yöntemi kullanılmayan diğer trafo merkezlerinde kontrol, saha ekipleri tarafindan yapılmakta ve arızalara geç müdahale edilmektedir.

Gömülü sistemler, günümüzde yaygın olarak kullanılan ve sadece belirli bir amacı gerçekleştirmesi için üretilen; kısıtlı bir işlemci gücü ve kaynağa (bellek, program hafizası vb.) sahip olan sistemler için kullanılan bir kavramdır. $\mathrm{Bu}$ sistemlerde gerçekleştirilmesi gereken işlemler için belirlenen süre hayati öneme sahiptir ve bu zaman kısitlamasına tamamen uyulması oldukça önemlidir. Örneğin; bir kavşakta bulunan trafik 1şıklarında meydana gelebilecek bir zaman sapması, farklı yollar için kısa süre de olsa aynı anda yeşil ışık yanması gibi, trafik kazasına sebep olabilmektedir. Bu sebeple bu tür sistemlerin gerçek zamanlı sistemler ile gerçekleştirilmesi gerekmektedir.

Gerçek zamanlı sistemlerde kullanılan işlemci genellikle tek çekirdekli olduğundan, görevler işlemci üzerinde sıra ile ve belirli süre kısıtlamasına tabi tutularak gerçekleştirilmektedir. $\mathrm{Bu}$ planlama, herhangi bir işletim sistemi kullanılmadan yapılabileceği gibi, bu amaç için tasarlanan Gerçek Zamanlı İşletim Sistemleri (GZISS) ile de yapılabilmektedir. GZIS ile görevler arasındaki geçiş belirli zaman aralıklarına bölünmekte ve çizelgeleme için ayrılan zaman azalmaktadır. Kullanılan GZIS'ne bağlı olarak gerekli zaman kısitlamalarına tam olarak uyulabilmektedir.

Önerilen sistemle trafo merkezlerinin kontrolü ve takibi Nesnelerin İnterneti (NI) teknolojisi yardımıyla uzaktan sağlanabilmektedir. $\mathrm{Bu}$ sayede trafo merkezlerinde meydana gelebilecek arızaların tek bir merkezden takibi ile arızaya erken müdahale imkanı sağlanabilmektedir. Böylece olası hayati ve maddi kayıpların boyutu da azalmaktadır. Ayrıca tasarlanan sistem sayesinde arıza sayısı, kesici ve ayırıcı gibi ekipmanlara ait açma ve kapama sayısı gibi istatistiki verilerin de toplanması Blynk uygulaması sayesinde mümkün olmaktadır. Bu sayede kesici ve ayırıcı gibi ekipmanlarının bakımları da planlanabilmekte, çok sayıda arıza yapan trafolar için gerekli önlemler alınabilmektedir. Önerilen sistemin ucuz olması ile sistem tüm trafo merkezlerine kurulabilmektedir. Ring sistemi oluşturan trafo merkezlerinde ise besleme aktarımının uzaktan sağlanması bu sayede mümkün olabilecektir. Önerilen sistemde belirlenen görevler arasında gerçek zamanlı geçiş için GZİS kullanılmıştır. $\mathrm{Bu}$ sayede görevlerin kesin bir süre içerisinde gerçekleşmesi sağlanmış ve daha sonra İnsan-Makine arayüzü gibi özelliklerin eklenmesine olanak sağlamıştır.

\section{Materyal ve Metot}

\subsection{Literatür Taraması}

Son y1llarda, gelişen teknolojiler ile birlikte Nİ teknolojisi çok fazla alanda kullanılmakta ve ilgi de giderek artmaktadır. NI, gelişen internet teknolojisi ile birlikte kullanım alanlarının ve ucuz teknolojilerin ortaya çıkması neticesinde yaygınlığının ve bulunabilirliğinin artması sayesinde trafo merkezlerinde kullanılması da gündeme gelmiştir. $\mathrm{Bu}$ amaçla önerilen sistemlerde (A H. Mohamad vd., 2018; Jamal vd., 2018; Kumar \& Ajitha, 2017; Mrs. A. P. Khandait vd., 2017; Pathania \& Aggarwal, 2019; Tarase \& Panchade, 2020) trafo merkezlerinin uzaktan kontrolü belli ölçülerde sağlanabilirken, çeşitli sensörlerden (gerilim, akım vb.) gelen veriler takip edilebilmektedir. (Dimpal vd., 2020; Kore vd., 2019; Pawar vd., 2017; Srivastava \& Tripathi, 2018) yapmış oldukları çalışmalarda meydana gelen arizalar e-posta veya SMS olarak iletilebilmektedir. Ayrıca, hastane gibi çok önemli yerlere elektrik verilmesinde öncelik tanıyan öneriler de bulunmaktadır (Hasan vd., 2019) ve bu öneriye göre trafo merkezindeki arıza giderildikten sonra elektrik enerjisinin dağıtımı öncelik sırasına göre yapılmaktadır. Trafo merkezlerinde toplanan veriler, uzak bilgisayarlardan izlenebileceği gibi, Android uygulaması ile telefondan da takip edilebilen sistemler geliştirilmiştir (Duttachowdhury vd., 2020). Bununla birlikte, trafo merkezlerinde bulunan kesici gibi bazı ekipmanların uzaktan kontrolünü yapan sistemler de önerilmiştir (Hossain vd., 2019).

Trafoların kontrol ve takibinin yapilmasında, yapay sinir ağları ve bulanık mantık gibi yapay zeka yöntemleri kullanan bazı uygulamalar geliştirilmiş̧ir (Khairnar vd., 2020; Žarković \& Stojković, 2017). (Cheng \& Wang, 2011; Emi vd., 2019; Rahman vd., 2017) yapmış oldukları çalışmalarında trafoda meydana gelen hataları hücresel ağ teknolojisi ile iletilmesini önermişlerdir. (Zhao vd., 2019) trafo merkezindeki çeşitli bilgilerin FPGA vasitasıyla toplanıp, ağ zaman protokolü ile saat bilgisi alınarak uzak bilgisayar ile senkronize eden bir sistem tasarlamışlardır. (Yaman \& Biçen, 2019) ise sensör değerlerinin kritik değerleri aşması neticesinde kullanıcıya bildirim gönderen bir uygulama önermişlerdir. (Thangiah vd., 2019), toplanan sensör verilerinin değerlendirilmesinde uç yapay zeka yöntemini kullanılmışlardır. Bununla birlikte, Raspberry Pi gibi ucuz ekipmanların kullanıldığı öneriler de mevcuttur (John vd., 2017). Bu sayede SCADA ve PLC gibi pahalı yöntemlere bir alternatif önerilmiştir. Mariprasath ve Kirubakaran trafonun durum kontrolünün termal görüntü ile yapılmasını önermişlerdir (Mariprasath \& Kirubakaran, 2018). Önerilen başka bir sistemde trafo merkezleri ile iletişim uydu üzerinden sağlanmıştır (Sohraby vd., 2018). Wang ve arkadaşları ise trafo merkezlerinde bulunan SF6 gibi tehlikeli gazların takibinin yapılmasının yanı sıra kamera ile uzaktan izlemenin yapilabileceği bir sistem önermişlerdir (Wang vd., 2013). Aynı sistemde sokak lambalarının kontrolü de sağlanmaktadır. Ayrıca; makine öğrenmesinin Nİ ile birlikte kullanıldığı sistem önerisi de bulunmaktadır (Zhang vd., 2020).

Gerçekleştirilen çalışma da ise ESP32 geliştirme kiti ile IOS ve Android üzerinde çalışabilen Blynk uygulaması üzerinden izleme ve kontrol yapılabilmektedir. Önerilen sistem ile yüksek gerilim durumlarına, yağ seviyesi arızası, yüksek sıcaklık ve aşırı yük veya akım gibi problemlere gerçek zamanlı müdahale imkanı sağlanmıştır. Daha önce kullanılan sistemlerde bulunan 
özelliklere artı olarak gerçek zamanlı işletim sisteminin kullanılması, öncelik mekanizması, Bluetooth gibi farkl1 haberleşme teknolojilerin eklenmesi, mobil haberleşme ve özellikle maliyeti emsallerine göre düşük bir çözüm önerisi olması sistemin öne çıkan özellikleridir. Önerilen sistemin nesnelerin internetinin uygulama alanlarından biri olan akıllı şehirlerde kullanılan akıllı lambalar ile entegre bir senaryo ile birlikte kullanılması da mümkündür.

\subsection{Sistem Mimarisi}

Elektriğin hayatımızdaki yeri ve önemi arttıkça, elektrik enerjisinin evlerimize ve işyerlerimize dağıtılmasından sorumlu trafo merkezlerinin takibi hayati önem arz etmektedir. Trafoların izlenmesi ve arızalara zamanında müdahale edilmesi hayati ve maddi kayıpların azalmasını hatta kimi durumlarda önlenmesini sağlamaktadır.Trafolar için tehlike arz eden aşağıdaki durumlara karşı önlem alınması gerekmektedir:

- Yüksek Gerilim: Şehir şebekesinde kullanılan trafolar genellikle $34,5 \mathrm{kV}$ giriş gerilimini $220 \mathrm{~V}$ ve $380 \mathrm{~V}$ AC'ye dönüştürmektedir. Her devre elemanı gibi trafoların da bir dayanım gerilimi bulunmaktadır. $\mathrm{Bu}$ dayanım geriliminin aşılması trafoların zarar görmesine neden olur. Modern çıkış hücrelerinde bu gerilimi takip edip kesiciyi açtıran bir işlemci ünite bulunmaktadır. Önerilen sistem bu sinyali alıp kesiciyi açtırdığı gibi internet üzerinden de bu bilgiyi yayınlamaktadır.

- Yağ Seviye Arızası: Trafolarda kullanılan soğutma yağının durumunu kontrol eden Buschollz rölesi üzerinden alınan bilgi ile kesici güç anahtarı açtırılmaktadır.

- Yüksek Sicaklık: Sicaklığın artması trafoyu ciddi şekilde tehdit etmektedir. Sicaklık sensöründen alınan veriye göre fan çalıştırılmakta, sıcaklığın aşırı artması ile kesici anahtarı açtırılarak trafonun daha fazla çalışması önlenmektedir.

- Aşırı Akım/Yük: Trafo merkezlerinde kullanılan trafoların besleyebileceği bir akım/yük miktarı bulunmaktadır. Kışın elektrikli sobaların, yazın da klimaların kullanılması sebebiyle trafoların karşılayabileceğinden daha fazla akım çekilebilmektedir. Trafo merkezlerinde bu akımı takip edip kesiciyi açtıran bir işlemci ünite bulunmaktadır. Önerilen sistem bu sinyali alıp kesiciyi açtırdığı gibi internet üzerinden bu bilgiyi de yayınlayarak arızaya müdahale edilmesini sağlamaktadır.

\subsubsection{Blok Diyagram}

Önerilen sistem mimarisi Resim. 1'de görülmektedir. Bu sistemde kontrol, ESP32 kiti ile sağlanmıştır. Trafo merkezlerinde kullanılan sensörlerden gelen bilgiye göre kesicinin kontrolü sağlanmıştır. Sensörlerden elde edilen veriler kablosuz internet aracılığıyla Blynk uygulamalarına gönderilmiştir. $\mathrm{Bu}$ sayede sistemin uzaktan takibi ve kontrolü mümkün kılınmıştır. Kablosuz ağ üzerinden alınan saat verisi ile sokak lambaları güneşin doğuş ve batış zamanlarına göre açılıp kapatılmıştır. Sıcaklık sensöründen alınan bilgiye göre fan aktif edilmekte, buna rağmen sicaklığın yükselmeye devam etmesi durumunda kesici açtırılarak trafo sargılarının daha fazla ısınıp yanmasına engel olunmaktadir.

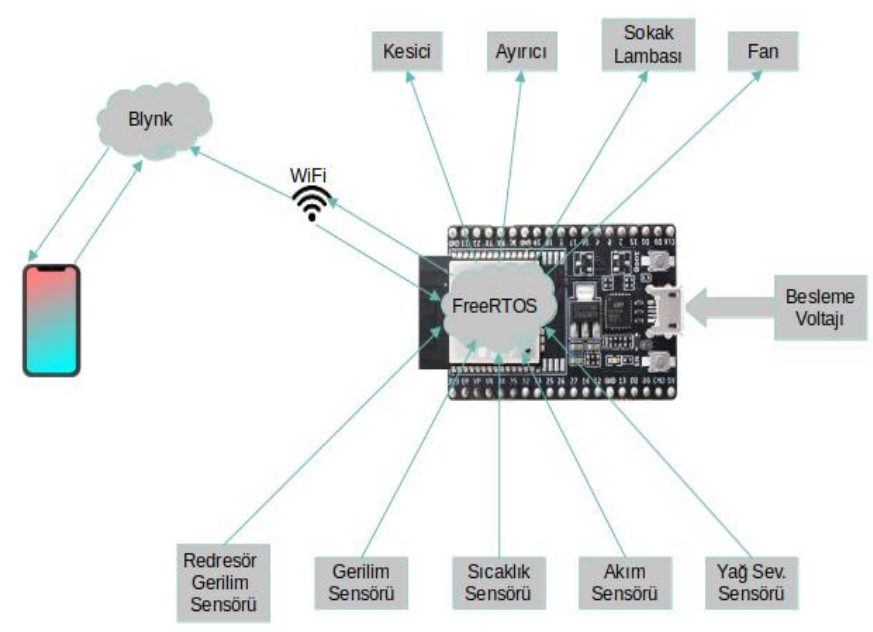

Resim.1 Blok diyagram.

\subsection{2 Çalışma Prensibi}

Sistemin kontrolü için ESP32 kiti kullanılmıştır ve veriler bu kit sayesinde Blynk platformuna gönderilmiştir. Sistemde sıcaklık sensörü ve redresör gerilimini simüle etmesi için potansiyometre kullanılmıştır. Okunan sıcaklık değerine göre fanın çalıştırılması ve aşırı yüksek sıcaklıklarda kesicinin açtırılması sağlanmıştır. Bununla birlikte redresör geriliminin belirli bir değer altına düşmesi ile kullanıcıya Blynk uygulaması üzerinden bilgi gönderilmesi sağlanmıştır. Diğer sensörler için ise trafo merkezlerinde standart olarak bulunduğu düşünülen sensörlerden gelen veriler lojik bilgi olarak değerlendirilmiştir. ESP32 kitinde kullanılan işlemcinin programlaması için Arduino IDE ve hızlı prototipleme için hazır kütüphaneler kullanılmıştır. Sistem yazılımı için kullanılan algoritmanın akış şeması Resim. 2'de görülmektedir. Akış şemasından görüleceği üzere yazılımda sensörler ve Blynk uygulamasından gelen komutlar takip edilmektedir. Kesici ve ayırıcı ekipmanlarının kontrolüne ait akış şeması ise Resim. 3'te görülmektedir.

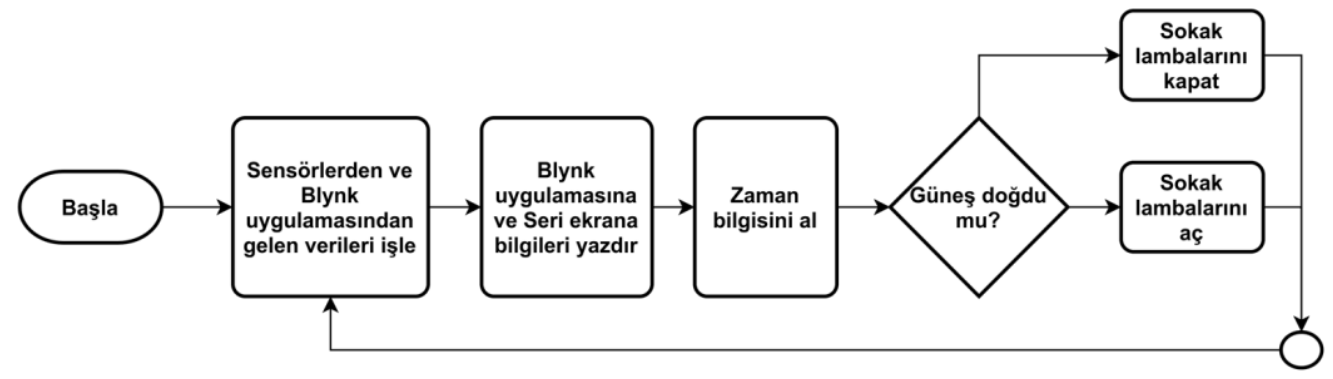

Resim.2 Uygulamaya ait akış şemast. 


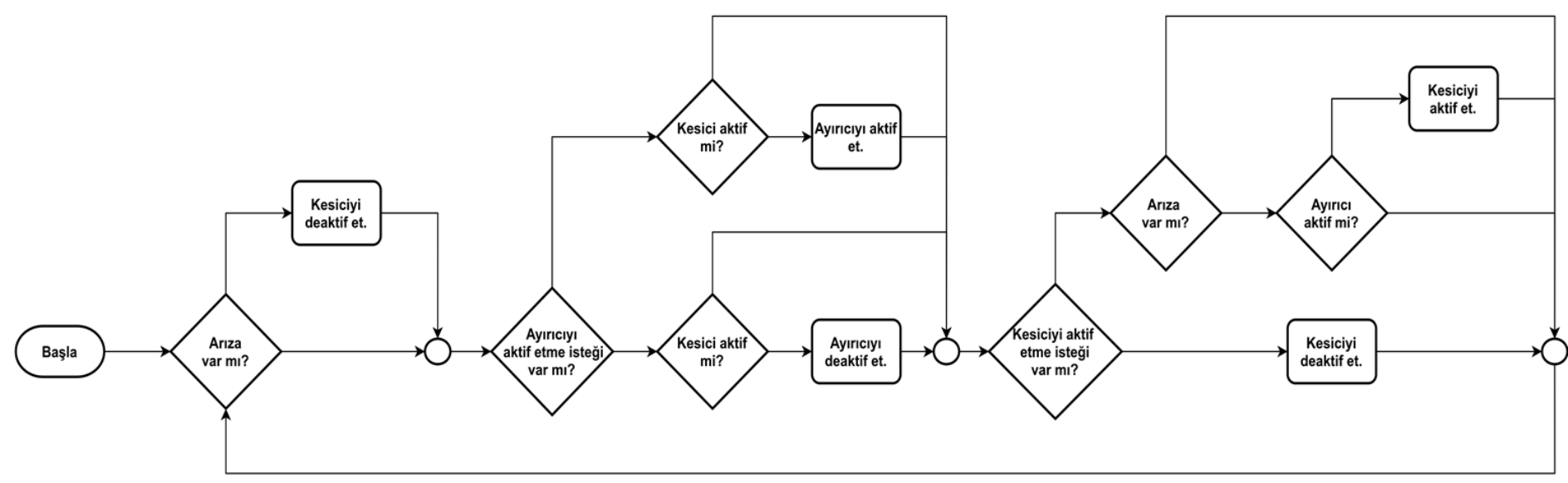

Resim.3 Kesici ve ayırıcı ekipmanlarının kontrolüne ait akış şeması.

Önerilen sistemde yapılan diğer işlemler şu şekildedir:

- Güneşin Doğuş ve Batış Zamanının Belirlenmesi: Güncel saat bilgisi ağ zaman protokolü ile alınarak güneşin doğuş ve batış zamanına göre sokak lambalarının çalıştırılması sağlanmıştır. Güneşin doğuş ve batış anını hesaplamak için kullanılan (Denklem 1) algoritmada (Sunrise/Sunset Algorithm) öncelikle bulunulan günün yılın hangi gününe denk geldiği belirlenmelidir. $\mathrm{Bu}$ amaçla, güncel saat bilgisinin yanı sıra ay ve yıl bilgisi de alınmaktadır. Daha sonra güneşin ortalama anomali hesabını yapabilmek için bulunulan boylam değeri saat cinsine dönüştürülmektedir. Ortalama anomali değeri ile, güneşin açısal uzaklığını hesaplamak için kullanılan sağ açıklık değerini hesaplayabilmek için güneşin gerçek boylam değeri hesaplanır. Böylece güneşin belirli bir zamanda belirtilen koordinat değerlerine göre konumu belirlenebilecektir. Bununla birlikte, güneşin gerçek boylam değeri, güneşin ekvatora göre olan konumunu ifade eden dik açıklık değerini hesaplamak için de kullanılmaktadır. Hesaplanan dik açıklık değeri ve güneş ışınları ile dikey yön arasındaki açıyı belirten zenit açısı kullanılarak yerel saat bilgisi açı cinsinden hesaplanmaktadır. Son olarak hesaplanan bu değer dakika cinsine, yerel ofset değeri de eklenerek güneşin doğuş ve batış zamanı belirlenebilmektedir.

$$
\omega_{\circ}=\frac{\left(-0.83^{\circ}\right)-\phi \times \delta}{\phi \times \delta}(1)
$$

- Kesici ve Ayırıcı Kontrolü: Trafo merkezlerinde bulunan kesici ve ayırıcıların uzaktan kontrolü Blynk uygulaması sayesinde yapılabilmektedir. Yazılan kodda ayırıcı

kapalı değilken kesicinin kapatılmasına engel olunmuştur. Aynı şekilde sistem enerjili iken (kesici kapalı durumda) ayırıcının açılması da önlenmiştir. Bu sayede ayırıcının yanlış şekilde çalıştırılmasının önüne geçilerek ömrü uzatılmıştır. Sistemde bir arıza olduğu sensörler tarafindan bildiriliyorsa kesicinin aktif edilerek trafonun enerjilendirilmesinin önüne geçilmiştir. Blynk uygulamasına ait ekran çıktıları Resim 4'te görülmektedir.

- Uzak İzleme: Trafo merkezinde bulunan sensörlerden alınan veriler Blynk uygulamasına gönderilmiştir. Böylece merkez uzaktan izlenebilmektedir. Bununla birlikte, sistemin kontrolü de yine aynı uygulama üzerinden yapılabilmektedir.
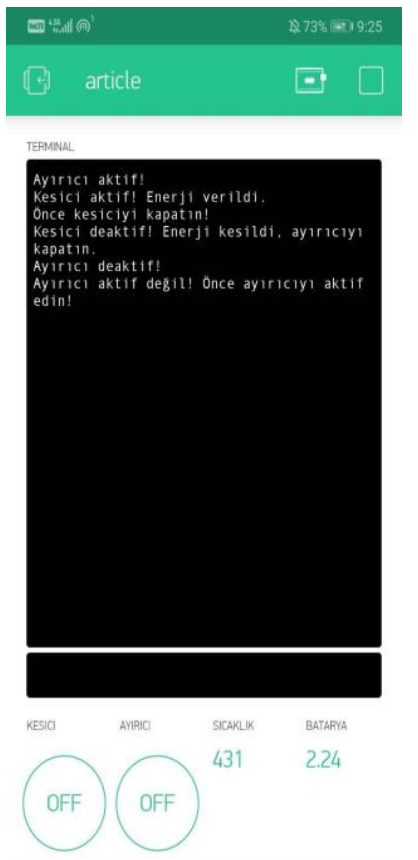
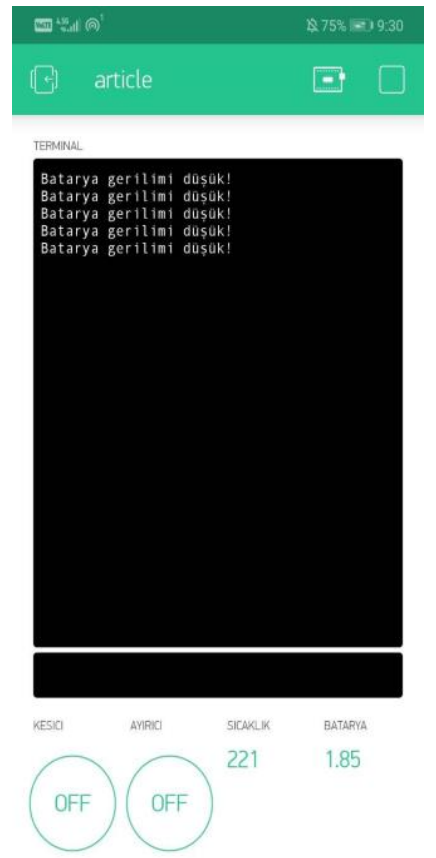
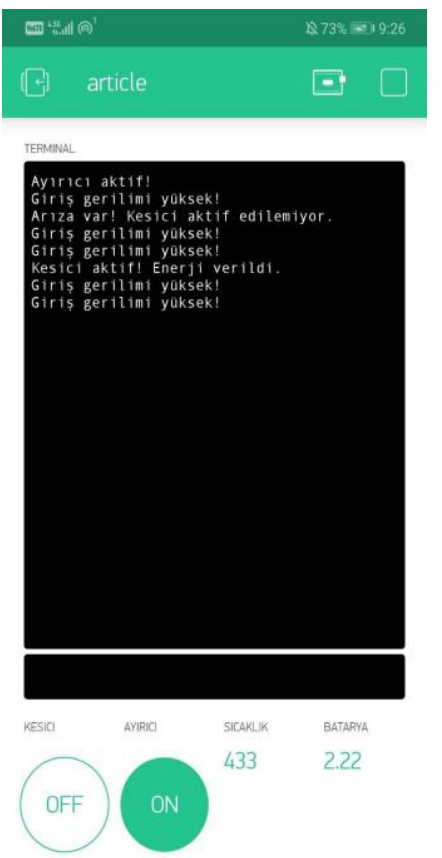
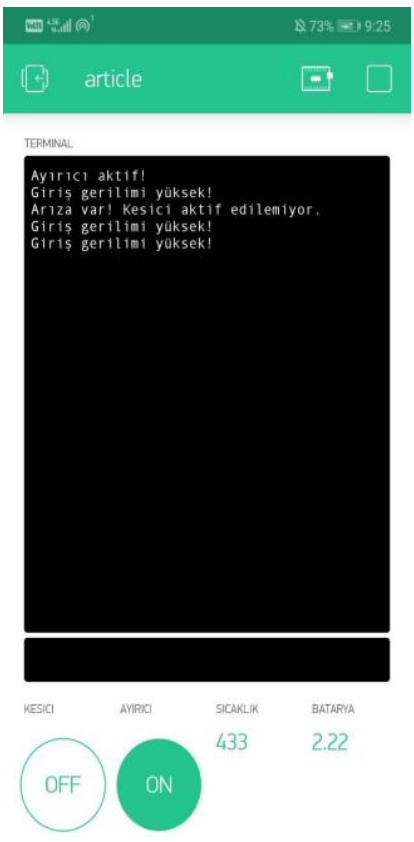

Resim.4 Blynk uygulamasına ait ekran görüntüleri. 


\section{Araştırma Sonuçları ve Tartışma}

Prototipin geliştirilmesinde kullanılan ESP32 kiti çift çekirdekli işlemciye sahip olması, Bluetooth ve WiFi çevre birimlerine sahip olması gibi özelliklerinin yanı sıra, Arduino IDE ile birlikte kullanılması neticesinde hızlı prototip geliştirmeye imkan tanımıştır. Kitin yaygın kullanıma sahip olması da hızlı geliştirme yapılmasında etkili olmuştur. Güneşin doğuş ve batış zamanının hesaplanmasında farklı algoritmalar kullanılmaktadır ("Sunrise Equation", 2021; US Department of Commerce). Ancak, Sakarya ili için en doğru sonucu veren algoritma ile yapılan hesaplamalarda gerçek zaman değerleri ile program tarafindan hesaplanan değerler arasında çok az bir sapma meydana geldiği görülmüştür. Gerçekleştirilen uygulamada bu değer tolere edilebilmektedir.

GZİS'nin açık kaynak kodlu olmasının getirdiği avantaj sayesinde gerekli modifikasyon işlemleri gerçekleştirilebilmiştir. Görevler arasında öncelik ataması yapılabilmesi özelliği ile önemli görevlerin önceliğinin arttırılması ile birlikte sistemin güvenliği arttırılmıştır. Bununla birlikte, Arduino IDE geliştirme ortamı ve hazır kütüphaneler her ne kadar doğru ve hızlı geliştirmeye olanak sağlasa da tam olarak gerçek zaman kısıtlarına uyulmasına zaman zaman engel olmaktadır. Bu nedenle, görevler arasındaki geçişlerde gecikme olmaması amacıyla GZİS kullanılmıştır.

Trafo merkezlerinde bulunan ayırıcı ve kesici ekipmanlarının uzaktan kontrolünün sağlanabilmesi sonucunda ring sisteminde bulunan merkezler arasındaki enerji aktarımı uzaktan kontrol edilebilmektedir. $\mathrm{Bu}$ merkezlerde kesici ve ayırıcının manevra işlemlerinin yapılmasını sağlayan redresör grubu gerilimi de uzaktan izlenebilmektedir. Bunun sonnucunda redresör grubunda tespit edilen bir arızaya hızlı bir şekilde müdahale edilebilmesi sayesinde trafo merkezinin erişilebilirliği arttırılmıştır.

\section{Sonuç}

Geliştirilen sistem, trafo merkezlerinin uzaktan kontrol ve izlenmesinde yaygın bir şekilde kullanılan SCADA sistemlerine göre daha az maliyetli ve gerçek zamanlı haberleşmeye imkan sağlayan, mobil teknolojiler gibi birçok yenilikçi yönü olan bir alternatif çalışma olmuştur. Prototipin geliştirilmesinde kullanılan gerçek zamanlı işletim sistemi ARM, Intel, Xilinx mikroişlemcileri başta olmak üzere birçok işlemci mimarisini desteklemektedir. $\mathrm{Bu}$ nedenle prototipin diğer mimarilere aktarılmasına olanak sağlamaktadır. Mobil uygulamalarda çalışabilmesi için Blynk uygulaması kullanılmıştır ve program üzerinden izleme ve kontrol yapılması sağlanmıştır. Prototipin geliştirilmesinde kullanılan araçlar, önerilen sistemin gelişime açık olmasını ve farklı sistemlere adapte edilebilmesini sağlamıştır. Böylece, önerilen sisteme İnsan-Makine arayüzü gibi eklenebilecek özelliklerin sayısı artmıştır. Bu nedenle, sistemin güvenliğini ve izlenmesini arttırabilecek eklentiler yapılması oldukça kolaylaşmıştır. Akıllı lambalar gibi birçok nesnelerin interneti uygulamasıyla önerilen sitemin birlikte kullanılması da mümkündür.

\section{Kaynakça}

A H. Mohamad, A., S. Mezaal, Y., \& F. Abdulkareem, S. (2018). Computerized power transformer monitoring based on internet of things. International Journal of Engineering \& Technology, 7(4), 2773.
Cheng, X., \& Wang, Y. (2011). The remote monitoring system of transformer fault based on The internet of Things. Proceedings of 2011 International Conference on Computer Science and Network Technology, 1, 84-87.

Dimpal, M. P., Mayank, P., Pratik, G., Pramod, K., \& Kshitija, D. (2020). Monitorıng And Controlling Of Transformer Using IoT. International Journal of Advance Research and Innovative Ideas in Education (IJARIIE), 6(5).

Duttachowdhury, D., Patil, V., Parab, A., Patel, R., \& Nikum, K. Transformer Monitoring and Control Using IoT. IOSR Journal of Engineering (IOSR-JEN) vol. 10, 40-43

Emi, P., Sivasankari, R., Kumar, P., Prabha, R., Jayageetha, J., \& Karhikeyan, A. (2019). Fault Detection in Transformer Using GSM Technology. 868-873.

Hasan, W. K. A., Alraddad, A., Ashour, A., Ran, Y., Alkelsh, M. A., \& Ajele, R. A. M. (2019). Design and Implementation Smart Transformer based on IoT. International Conference on Computing, Electronics \& Communications Engineering, 16-21.

Hossain, Md. S., Rahman, M., Sarker, Md. T., Haque, Md. E., \& Jahid, A. (2019). A smart IoT based system for monitoring and controlling the sub-station equipment. Internet of Things, 7, 100085 .

Jamal, H., Nadeem Khan, M. F., Anjum, A., \& Janjua, M. K. (2018). Thermal Monitoring and Protection for Distribution Transformer Under Residential Loading Using Internet of Things. 2018 IEEE Global Conference on Internet of Things (GCIoT), 1-6.

John, A., Varghese, R., Krishnan, S. S., Thomas, S., Swayambu, T. A., \& Thasneem, P. (2017). Automation of $11 \mathrm{kv}$ substation using raspberry pi. 2017 International Conference on Circuit ,Power and Computing Technologies (ICCPCT), $1-5$.

Khairnar, V., Kolhe, L., Bhagat, S., Sahu, R., Kumar, A., \& Shaikh, S. (2020). Industrial Automation of Process for Transformer Monitoring System Using IoT Analytics. Içinde G. Ranganathan, J. Chen, \& Á. Rocha (Ed.), Inventive Communication and Computational Technologies (ss. 11911200). Springer.

Kore, P., Ambare, V., Dalne, A., Amane, G., Kapse, S., \& Bhavarkar, S. (2019). IoT Based Distribution Transformer Monitoring and Controlling System. Journal of Research and Advancement in Electrical Engineering, 2(1).

Kumar, T. A., \& Ajitha, A. (2017). Development of IoT based solution for monitoring and controlling of distribution transformers. 2017 International Conference on Intelligent Computing, Instrumentation and Control Technologies (ICICICT), 1457-1461.

Mariprasath, T., \& Kirubakaran, V. (2018). A real time study on condition monitoring of distribution transformer using thermal imager. Infrared Physics \& Technology, 90, 78-86.

Mrs. A. P. Khandait, Swapnil Kadaskar, Girish Thakare, \& Priyadarshini college of Engineering,nagpur. (2017). Real Time Monitoring of Transformer using IOT. International Journal of Engineering Research And, V6(03), IJERTV6IS030200.

Pathania, S., \& Aggarwal, S. K. (2019). IoT Based Distribution Transformer Health Monitoring System [Thesis]. http://tudr.thapar.edu:8080/jspui/handle/10266/5788, Erişim tarihi 20 Eylül 2021

Pawar, R. R., Wagh, P. A., \& Deosarkar, S. B. (2017). Distribution transformer monitoring system using Internet of 
Things (IoT). 2017 International Conference on Computational Intelligence in Data Science(ICCIDS), 1-4.

Peharda, D., Ivanković, I., \& Jaman, N. (2017). Using Data from SCADA for Centralized Transformer Monitoring Applications. Procedia Engineering, 202, 65-75.

Rahman, S., Dey, S. K., Bhawmick, B. K., \& Das, N. K. (2017). Design and implementation of real time transformer health monitoring system using GSM technology. 2017 International Conference on Electrical, Computer and Communication Engineering (ECCE), 258-261.

SCADA. https://www.sedas.com/trtr/DagitimHizmetleri/Pages/Scada.aspx, Erişim tarihi 14 May1s 2021,

Sohraby, K., Minoli, D., Occhiogrosso, B., \& Wang, W. (2018). A Review of Wireless and Satellite-Based M2M/IoT Services in Support of Smart Grids. Mobile Networks and Applications, 23(4), 881-895.

Srivastava, D., \& Tripathi, M. M. (2018). Transformer Health Monitoring System Using Internet of Things. 2018 2nd IEEE International Conference on Power Electronics, Intelligent Control and Energy Systems (ICPEICES), 903-908.

Sunrise equation. (2021). Wikipedia. https://en.wikipedia.org/w/index.php?title=Sunrise_equation \&oldid=1023027239, Erişim tarihi 14 Mayıs 2021

Sunrise/Sunset

Algorithm. https://edwilliams.org/sunrise_sunset_algorithm.htm, Erişim tarihi 14 May1s 2021,

Tarase, K. B., \& Panchade, V. M. (2020). Monitoring amp; Controlling of Substation Using IoT in Distribution Power Grid. 2020 5th International Conference on Devices, Circuits and Systems (ICDCS), 66-70.

Thangiah, L., Ramanathan, C., \& Chodisetty, L. S. (2019). Distribution Transformer Condition Monitoring based on
Edge Intelligence for Industrial IoT. 2019 IEEE 5th World Forum on Internet of Things (WF-IoT), 733-736.

Tom, R. J., \& Sankaranarayanan, S. (2017). IoT based SCADA integrated with Fog for power distribution automation. 2017 12th Iberian Conference on Information Systems and Technologies (CISTI), 1-4.

US Department of Commerce, N. ESRL Global Monitoring Laboratory-Global Radiation and Aerosols., https://gml.noaa.gov/grad/solcalc/calcdetails.html,_Erişim tarihi 20 Eylül 2021

Wang, J. H., Li, X. Z., Lu, R. T., \& Sun, F. J. (2013). Internet of Things Based Auxiliary Control System for Smart Substation. Advanced Materials Research, 614-615, 17901795.

Wester, C., Engelman, N., Smith, T., Odetunde, K., Anderson, B., \& Reilly, J. (2015). The role of the SCADA RTU in today's substation. 2015 68th Annual Conference for Protective Relay Engineers, 622-628.

Yaman, O., \& Biçen, Y. (2019). An Internet of Things (IoT) based Monitoring System for Oil-immersed Transformers. Balkan Journal of Electrical and Computer Engineering, 7(3), 226234.

Žarković, M., \& Stojković, Z. (2017). Analysis of artificial intelligence expert systems for power transformer condition monitoring and diagnostics. Electric Power Systems Research, 149, 125-136.

Zhang, C., He, Y., Du, B., Yuan, L., Li, B., \& Jiang, S. (2020). Transformer fault diagnosis method using IoT based monitoring system and ensemble machine learning. Future Generation Computer Systems, 108, 533-545.

Zhao, L., Brandao Machado Matsuo, I., Zhou, Y., \& Lee, W.-J. (2019). Design of an Industrial IoT-Based Monitoring System for Power Substations. IEEE Transactions on Industry Applications, 55(6), 5666-5674. 